\title{
Transformation of medical education in uncertainty
} KJME

Young-Mee Lee

Department of Medical Education, Korea University College of Medicine, Seoul, Korea

While the coronavirus disease 2019 (COVID-19) pandemic has generated unprecedented panic and fear worldwide, the devotion of medical personnel in every country has inspired a sense of solidarity among public healthcare professionals. Their dedication in treating their patients is based on a sense of duty and professionalism that is deeply rooted in their hearts. As we cannot be certain about the frequency of such disasters in the coming years, the role of medical education in nurturing future doctors who can overcome unpredictable real-life situations based on the firm foundations of the scientific approach and professionalism has become ever so important.

To respond proactively to a rapidly changing society, medical education attempted many changes, including information technology-based education, over the last couple of decades. However, since it mainly relies on teaching and learning using face to face interactions, the prevailing norm of social distancing has resulted in several challenges. Although online lectures faced difficulties such as technical issues and unfamiliarity among students and professors during their initial implementation, the new system has since stabilized and the learners have rapidly grown accustomed to it. However, other modes of education, such as experiments, small-group discussions, simulations, and clerkships rotations can only be effectively operationalized to reach educational goals through real-time face to face classes. Especially in the case of clerkship rotations, the human-to-human interaction is an integral and irreplaceable experience. As COVID-19 continues to spread throughout society, it has even affected teaching hospitals, resulting in the suspension of clinical rotations. The assessment of clinical performance examinations (CPX) and objective structured clinical examinations (OSCE) is only effectively executed on-site. While the postponement or cancellation of such clinical assessments at the individual school level may produce several problems, if the current situation persists, highstakes examinations such as a medical licensure examination that uses CPX/OSCE can be affected. If we are not adequately prepared, there might be a significant shortage in the supply of medical personnel next year.

Facing new challenges every day, medical schools and
Received: April 2, 2020 • Revised: April 8, 2020 • Accepted: April 8, 2020 Corresponding Author: Young-Mee Lee (https://orcid.org/0000-0002-4685-9465) Department of Medical Education, Korea University College of Medicine, 73 Goryeodae-ro, Seongbuk-gu, Seoul 02841, Korea

Tel: +82.2.2286.1098 email: ymleehj@korea.ac.kr
Korean J Med Educ 2020 Jun; 32(2): 89-90.

https://doi.org/10.3946/kjme.2020.155

eISSN: 2005-7288

(C) The Korean Society of Medical Education. All rights reserved. This is an open-access article distributed under the terms of the Creative Commons Attribution Non-Commercial License (http:// creativecommons.org/licenses/by-nc/3.0/), which permits unrestricted non-commercial use, distribution, and reproduction in any medium, provided the original work is properly cited. 
all stakeholders in medical education are overcoming the current situation through their creativity and collective intelligence. This may be the first time in history when medical educators around the world have come together to confront a single common interest. If medical schools or medical educators can share how they are coping with the issues that are placing the various dimensions of medical education, it may reduce unnecessary fears or concerns among those who will face similar problems in the near future and help them to manage these challenges in a more wise and flexible manner.

Accordingly, the Korean Journal of Medical Education (KJME) will introduce a special section, called "Medical education in the age of uncertainty," from the 2020 June issue in order to share the collective experience of how these tough times can be utilized to transform medical education. We wish to hear from students, professors, and schools alike; we are willing to receive manuscripts regarding all fields of medical education. The authors submitting to this new section can use the KJME's existing guidelines for short communication, commentaries/opinions, and personal views. All submitted manuscripts will be peer reviewed, evaluated, and approved for publication as before. We will use our standard criteria of originality, clarity, and significance to decide which manuscripts to include. To help our readers around the world to collectively solve various issues, the rapid dissemination of information on how we are addressing the diverse challenges emerging from the COVID-19 pandemic is our primary concern. Therefore, we are planning to review and evaluate these submissions as swiftly as we can.

We invite you to submit your manuscripts to "Medical education in the age of uncertainty" to share your knowledge and experience as stakeholders in medical education so that we can collectively prepare for future uncertainties.

\section{ORCID:}

Young-Mee Lee: https://orcid.org/0000-0002-4685-9465

Acknowledgements: None.

Funding: None.

Conflicts of interest: No potential conflict of interest relevant to this article was reported.

Author contributions: All work was done by Young-Mee Lee. 